\title{
Factors Associated with Caregiver Burden in Dementia: 1-Year Follow-Up Study
}

\author{
Sang Hong Shim ${ }^{1}$, Hyo Shin Kang ${ }^{1}$, Ji Hae Kim², and Doh Kwan Kim¹,2凶 \\ ${ }^{1}$ Center for Clinical Research, Samsung Biomedical Research Institute, Seoul, Republic of Korea \\ ${ }^{2}$ Department of Psychiatry, Samsung Medical Center, Sungkyunkwan University School of Medicine, Seoul, Republic of Korea
}

Objective Dementia symptoms (cognitive function, daily-living function, and neuropsychiatric symptoms) become more serious over time, which is likely to increase caregiver burden. The aim of this study is to investigate which dementia-related symptoms, and how the progression of these symptoms, have influenced caregiver burden during a 1-year follow-up assessment.

Methods A total of 110 patients with dementia were assessed for their cognitive function, daily-living function, and neuropsychiatric symptoms. Caregivers were assessed for their caregiver burden. Bivariate analyses were conducted between caregiver burden and dementia patients' symptoms, in order to examine which particular symptoms were significantly associated with caregiver burden at the baseline. A multiple regression analysis was then conducted with each significantly associated variable with a view to identifying determinants, influencing caregiver burden. Additionally, bivariate analyses were conducted between the changes in caregiver burden and the changes in patients' symptoms, to investigate which patient variable could best describe caregiver burden from baseline to the 1-year follow-up. A multiple regression analysis was conducted with each significantly-associated change in symptom, in order to identify determinants that influence a change in caregiver burden.

Results Neuropsychiatric symptoms, such as irritability, aberrant motor-behavior, delusions and disinhibition were found to be significant predictors of caregiver burden at baseline, according to multiple regression analysis. In addition, changes in neuropsychiatric symptoms, such as delusions, agitation and memory-related functioning in daily-living significantly predict a change in caregiver burden.

Conclusion Our results demonstrate that neuropsychiatric symptoms and memory impairment in daily-living functions are significant predictors of an increase in caregiver burden.

Psychiatry Investig 2016;13(1):43-49

Key Words Dementia, Caregiver burden, Neuropsychiatric symptoms, Daily-living function, Cognitive function.

\section{INTRODUCTION}

Caregivers of dementia patients play an important role in managing the progress of dementia. This care carries a high burden when a loved-one develops dementia or a condition associated with dependency. ${ }^{1}$ Caregiver burdens include chronic fatigue, a sense of frustration, guilt, depressive symptoms, excessive distress, and loss of control over the caregiving situation. ${ }^{2,3}$ As a result, caregiver neglect can negatively influence the progression of dementia symptoms in patients, or increase the

Received: November 22, 2014 Revised: March 5, 2015

Accepted: March 28, 2015 Available online: November 20, 2015

$\triangle$ Correspondence: Doh Kwan Kim, MD, PhD

Department of Psychiatry, Samsung Medical Center, Sungkyunkwan University School of Medicine, 81 Irwon-ro, Gangnam-gu, Seoul 06351, Republic of Korea

Tel: +82-2-3410-3582, Fax: +82-2-3410-0941, E-mail: paulkim@skku.edu

(a) This is an Open Access article distributed under the terms of the Creative Commons Attribution Non-Commercial License (http://creativecommons.org/licenses/by$\mathrm{nc} / 3.0$ ) which permits unrestricted non-commercial use, distribution, and reproduction in any medium, provided the original work is properly cited. chance that the patient will require care services and facilities at an earlier stage. ${ }^{4,5}$ Therefore, caregiver burden is an important factor for the quality of life of both the caregiver and patient.

Previous studies have investigated important patient factors associated with caregiver burden, including cognitive function, activities of daily-living, and behavioral and psychological symptoms. ${ }^{1,6-9}$ The majority of these studies found that neuropsychiatric symptoms, such as anxiety, agitation, aggressive behavior, and disinhibition, are more closely related to caregiver burden than other symptoms, such as lower cognitive function or limitations in the activities of daily-living. ${ }^{8-10}$ However, inconsistent results have been reported in relation to whether cognitive deterioration of dementia patients is associated with increased caregiver burden. Certain studies have reported that lower cognitive function is associated with increased caregiver burden, ${ }^{6,11}$ whereas others have found that cognitive function itself does not affect caregiver burden., ${ }^{9,12}$ There are conflicting results on the relationship between the daily-living 
functioning of patients and caregiver burden.1,13 Some consistent results indicate that increased disturbance of neuropsychiatric symptoms is associated with caregiver burden, whereas both cognitive function and daily activity function show conflicting results. ${ }^{14}$

Although there are many conflicting studies, the relationship between caregiver burden and other patient functions and symptoms (deteriorated cognitive function, limited daily activity function, and neuropsychiatric symptoms) has only been investigated using a total score for each measurement. ${ }^{6,15}$ A simple total score analysis may affect the results, due to the fact that certain factors are very influential. Mini-Mental State Examination (MMSE), which is a measure of cognitive function, can be separated into six components. ${ }^{16}$ Additionally, several factors can be extracted from activities of daily-living function scales, such as the Seoul-Activities of Daily Living (S-ADL) $)^{17}$ and the Seoul-Instrumental Activities of Daily Living (SIADL). ${ }^{18}$ Therefore, it is necessary to examine whether there is a relationship between caregiver burden and the extracted detailed factors from the S-ADL and the S-IADL, as well as from the six Korean MMSE (K-MMSE) components. The relationship between caregiver burden and detailed patient symptoms or functions can be identified through each measurement.

Dementia symptoms can become more serious over time, and the progression of the severity of symptoms is likely to affect caregiver burden. ${ }^{14}$ Recently, Mohamed et al., ${ }^{15}$ conducted a follow-up study investigating the relationship between the progression of dementia symptoms and caregiver burden. Their results show that changes in caregiver burden were significantly correlated with changes in neuropsychiatric symptoms (positively) and daily-living functions (negatively). However, changes in caregiver burden were only weakly associated with cognitive function. In addition, it was shown that changes in neuropsychiatric symptoms could significantly predict changes in caregiver burden, better than could daily-living and cognitive functions. Nevertheless, the previous study ${ }^{15}$ was conducted within a short period (6-month follow-up) to identify detailed changes in caregiver burden and patient symptoms, thus it was limited to a clinical setting for persistent intervention and the assessment of dementia patients and their caregivers.

As a result of the aforementioned discrepancies in previous studies, we conducted the present study with the aim of examining the relationships between caregiver burden and patient variables (cognitive function, daily-living function, and neuropsychiatric symptoms) using baseline and 1-year follow-up data. Here, we also examine the relationship between the detailed factors of several measures of patient symptoms or functions, and caregiver burden. Furthermore, we examine the predicted change in caregiver burden through changes in patient variables.

\section{METHODS}

\section{Participants}

Data from 491 patients and their caregivers, who visited the Samsung Medical Center from March 2002 to April 2012, were selected. All patients met the dementia criteria of the Diagnostic and Statistical Manual of Mental Disorders, Fourth Edition ${ }^{19}$ and the National Institute of Neurological and Communicative Disorders and Stroke/Alzheimer's Disease and Related Disorders Association Work Group. ${ }^{20}$ A caregiver was defined as a person who lived with the patient, or who would most likely provide care if needed..$^{21}$ Of the 491 eligible patients, 344 patients were lost to non-follow-up at 1-year. Thirty-seven caregivers refused to complete a questionnaire [i.e., Zarit Burden Interview (ZBI)] assessing caregiver burden. Thus, data from 110 patients were used in the analysis. This study was approved by the Institutional Review Board of the Samsung Medical Center, and signed informed consent was obtained from both caregivers and patients.

\section{Measures}

\section{Dementia severity}

Dementia severity of patients at baseline was assessed with the clinical dementia rating $(\mathrm{CDR})$, a standardized global rating of dementia severity based on all available information about a patient. ${ }^{22} \mathrm{CDR}$ had a five-point scale ranging from 0 to 3. A higher CDR score indicates a more severe impairment: 0 (no cognitive impairment), 0.5 (very mild dementia), 1 (mild dementia), 2 (moderate dementia), and 3 (severe dementia).

\section{Cognitive function}

Cognitive function was assessed using the Korean MiniMental State Examination (K-MMSE), ${ }^{16,23}$ a brief 30-item measure of global cognitive ability. The K-MMSE is comprised of six components: time orientation (5 points), place orientation (5 points), registration (5 points), attention/calculation (5 points), recall (5 points), and language/visual construction (5 points). The K-MMSE had a score ranging from 0 to 30 .

\section{Daily function}

Daily function was assessed with the Seoul-Activities of Daily Living (S-ADL) ${ }^{17}$ and the Seoul-Instrumental Activities of Daily Living (S-IADL). ${ }^{18}$ The S-ADL was developed to assess an elderly individual's basic ability to perform activities of daily-living. It has 12 items (controlling bowels, controlling bladder, personal hygiene, using the toilet, feeding, transfer from floor to chair, walking indoors, dressing, ascending and descending stairs, bathing, left alone, and putting on shoes) on a three-point Likert scale ranging from 0 to 24 , with high- 
er scores indicating more dysfunctions. The S-IADL was also developed to assess an elderly individual's instrumental ability to perform activities of daily-living. The S-IADL has 15 items (using the telephone, shopping, preparing food/cooking, household chores, using transportation, walking outdoors, taking medications, managing finances, grooming, using household appliances, managing belongings, unlocking and closing an entrance door, keeping appointments, talking about recent events, and leisure/hobby activities) to evaluate functional abilities of daily-living. It has a four-point Likert scale ranging from 0 to 45 , with higher scores indicating more dysfunctions.

\section{Neuropsychiatric symptoms}

Neuropsychiatric symptoms were assessed using the Korean version of the Neuropsychiatric Inventory (K-NPI). ${ }^{24,25}$ The K-NPI can evaluate both frequency and severity of 12 neuropsychiatric symptoms (delusions, hallucinations, agitation, depression, anxiety, euphoria, apathy, disinhibition, irritability, aberrant motor behavior, sleep, and appetite). The KNPI score ranges from 1 to 12 for each composite domain, with higher scores indicating stronger symptoms. The NPI has previously demonstrated proper validity and reliability, ${ }^{25}$ and the reliability and validity of the Korean version of the NPI have also been established. ${ }^{24}$

\section{Caregiver burden}

Caregiver burden was measured by the Korean translation of the Zarit Burden Interview (ZBI), ${ }^{5,26}$ a widely used tool to measure perceived caregiver burden (e.g., "Do you feel you should be doing more for your relative?"). It has 22 items on a five-point Likert scale ranging from 1 (never) to 5 (nearly always). Total scale scores range from 22 to 110, with higher scores indicating greater caregiver burden.

\section{Study design and statistical analyses}

Baseline and 1-year follow-up data were gathered for caregiver burden (ZBI) and patient variables (K-MMSE, S-ADL, $\mathrm{S}$-IADL, and K-NPI). Factor analyses were conducted at baseline to extract the detailed S-ADL and S-IADL factors. Scores were calculated for each of the six K-MMSE components to determine whether there was any relationship between caregiver burden and patient variables (extracted detailed factors from the S-ADL and S-IADL, and six components of the KMMSE). Bivariate analyses were conducted between the total ZBI score and patient variables (K-MMSE, S-ADL, S-IADL, K-NPI, six components of the MMSE, and extracted detailed factors from the S-ADL and S-IADL) to examine which patient variables can best describe caregiver burden. A multiple regression analysis was subsequently conducted with each variable that was significantly correlated with the ZBI, to iden- tify determinants influencing caregiver burden. Bivariate analyses were also conducted between changes in the ZBI total score, and changes in the scores of the aforementioned patient variables. The change scores were obtained by subtracting baseline scores from the scores of the 1-year follow up. Subsequently, in order to determine which patient variable could best describe, and predict the change in, caregiver burden, a multiple regression analysis was conducted with change scores of each variable that significantly correlated with changes on the ZBI. Statistical analyses were carried out using SPSS ver. 19.0 (SPSS Inc., Chicago, IL, USA), and a result was considered statistically significant with a p value of less than 0.05 .

\section{RESULTS}

\section{Participant characteristics}

Mean age, gender ratio, years of education, ZBI, K-MMSE, S-ADL, S-IADL, and K-NPI scores of participants are summarized in Table 1. 68 (61.8\%) participants have Alzheimer's disease, 7 (6.4\%) have vascular dementia, and 35 (31.8\%) have other types of dementia including mixed dementia. CDR was rated at 0.5 for 18 participants (16.4\%), at 1 for 52 participants (47.3\%), at 2 for 35 participants (31.8\%), and at 3 for 5 partici-

Table 1. Demographic data and clinical characteristics $(\mathrm{N}=110)$

\begin{tabular}{|c|c|c|c|}
\hline & Number (\%) & \multicolumn{2}{|c|}{$\mathrm{M}(\mathrm{SD})$} \\
\hline Age (year) & & \multicolumn{2}{|c|}{$74.46(8.85)$} \\
\hline Education (year) & & \multicolumn{2}{|c|}{$8.18(4.74)$} \\
\hline Sex (\% female) & $83(75.5)$ & & \\
\hline \multicolumn{4}{|l|}{ Diagnosis } \\
\hline $\mathrm{AD}$ & $68(61.8)$ & & \\
\hline VD & $7(6.4)$ & & \\
\hline Other & $35(31.8)$ & & \\
\hline \multicolumn{4}{|l|}{ CDR } \\
\hline 0.5 & $18(16.4)$ & & \\
\hline 1.0 & $52(47.3)$ & & \\
\hline 2.0 & $35(31.8)$ & & \\
\hline \multirow[t]{2}{*}{3.0} & $5(4.5)$ & & \\
\hline & & (Baseline) & (1-year follow-up) \\
\hline ZBI & & $26.49(15.48)$ & $31.98(18.64)$ \\
\hline K-MMSE & & $16.26(5.48)$ & $13.65(6.51)$ \\
\hline S-ADL & & $3.26(4.40)$ & $6.05(6.30)$ \\
\hline S-IADL & & $24.98(9.86)$ & $29.89(9.88)$ \\
\hline K-NPI & & $12.85(9.36)$ & $14.69(11.50)$ \\
\hline
\end{tabular}

AD: Alzheimer's disease, VD: vascular dementia, CDR: Clinical Dementia Rating, ZBI: Zarit Burden Interview, K-MMSE: Korean version-Mini Mental State Examination, S-ADL: Seoul-Activities of Daily Living, S-IADL: Seoul-Instrumental Activities of Daily Living, K-NPI: Korean version-Neuropsychiatric Inventory, M: mean, SD: standard deviation 
pants (4.5\%). No significant correlation was observed between caregiver burden and age $(\mathrm{r}=-0.10, \mathrm{p}=\mathrm{n} . \mathrm{s}$.) or years of education $(\mathrm{r}=-0.02, \mathrm{p}=\mathrm{n} . \mathrm{s}$.). No significant difference was observed between caregiver burden and gender ratio $[\mathrm{F}(1,108)=0.13$, $\mathrm{p}=$ n.s.].

\section{Factor analyses}

We clarified the relationship between the S-ADL factors or the S-IADL factors and the ZBI total scores. Therefore, an exploratory factor analysis was conducted separately with the SADL and the S-IADL scores. Firstly, a maximum likelihood factoring method was used to extract factors from the S-ADL using the Promax (Kappa=4) rotation method. The results suggest that a three-factor solution was optimal. This was confirmed by a scree plot that revealed a clear break after the third factor. The first factor was comprised of items 3, 4, 5, 8, 10, 11, and 12 (Eigen value=5.92), which were related to "self-care/ hygiene". Items 6,7 , and 8 were included as the second factor (Eigen value $=1.60$ ), which represented "ambulation". The third factor included items 1 and 2 regarding "toilet use" (Eigen value $=1.10$ ). The three factors explained $49.32 \%$ of the total variance (Supplementary Table 1 in the online-only Data Supplement).

Two S-IADL factors were extracted based on the scree plot. Items $1,2,3,4,5,6,8,9,10,12$, and 14 were included in the first factor (Eigen value=7.04), whereas items 7, 11, 13, and 14 were included in the second factor (Eigen value=1.36). The first factor was called "non-memory-related functioning" related to "making phone calls", "using transportation", and "grooming". The second factor was called "memory-related functioning" including "keeping appointments", "talking about recent events", and "taking medications" (Supplementary Table 2 in the online-only Data Supplement).

\section{Bivariate analyses}

Bivariate analyses were carried out to investigate the relationships between caregiver burden and three patient variables [cognitive function, daily living function, and neuropsychiatric symptoms (Table 2)]. The total ZBI and the total K-NPI scores were positively associated $(\mathrm{r}=0.37, \mathrm{p}<0.001)$. Additionally, the correlation between the ZBI score and the total K-MMSE score and its six components were analyzed. As a result, the total K-MMSE score was marginally negatively correlated with the ZBI total score $(\mathrm{r}=-0.19, \mathrm{p}=0.05)$. The $\mathrm{ZBI}$ total score was negatively associated with "language and visual construction" $(\mathrm{r}=-0.20, \mathrm{p}<0.05)$.

The correlation analysis among the ZBI score, the total SADL score, and its three factors revealed a significant correlation between the ZBI score and self-care/hygiene ( $r=-0.20$, $\mathrm{p}<0.05)$. The same analysis was performed for the ZBI and the
S-IADL score and its two factors. The total S-IADL score and its two factors were significantly correlated with the ZBI (total S-IADL: $r=0.26 ; \mathrm{p}<0.01$; "non-memory-related functioning": $\mathrm{r}=0.28, \mathrm{p}<0.01$; "memory-related functioning": $\mathrm{r}=0.20, \mathrm{p}<0.05)$.

The relationships between the ZBI and the detailed behavioral problems that are linked to caregiver burden were examined. The total ZBI score was positively correlated with K-NPIdelusions $(\mathrm{r}=0.23, \mathrm{p}<0.05), \mathrm{K}-\mathrm{NPI}$-anxiety $(\mathrm{r}=0.24, \mathrm{p}<0.05)$, K-NPI-euphoria $(\mathrm{r}=0.21, \mathrm{p}<0.05), \mathrm{K}-\mathrm{NPI}$-disinhibition $(\mathrm{r}=0.26$, $\mathrm{p}<0.01)$, K-NPI-irritability $(\mathrm{r}=0.26, \mathrm{p}<0.01)$, and K-NPI-aberrant motor behavior $(\mathrm{r}=0.26, \mathrm{p}<0.01)$.

Correlation analysis was carried out to investigate whether there is a relationship between changes in the ZBI total score and changes in behavioral problems in dementia patients from baseline to the 1-year follow-up (Table 2). Changes in the ZBI score were significantly correlated with the total K-NPI score $(\mathrm{r}=0.42, \mathrm{p}<0.001)$, "self-care/hygiene" $(\mathrm{r}=0.22, \mathrm{p}<0.05)$, the total S-IADL score $(\mathrm{r}=0.25, \mathrm{p}<0.01)$, and the S-IADL "memoryrelated functioning" factor $(\mathrm{r}=0.31, \mathrm{p}=0.001)$.

Finally, the correlation between changes in caregiver burden and changes in behavioral problems was examined. The ZBI total score was significantly correlated with K-NPI-delusions ( $\mathrm{r}=0.37, \mathrm{p}<0.001)$, K-NPI-irritability $(\mathrm{r}=0.34, \mathrm{p}<0.001)$, K-NPIdepression $(r=0.26, p<0.01)$, and K-NPI-sleep $(r=0.27, p<0.01)$.

Table 2. Bivariate correlations between caregiver burden and other variables at baseline and bivariate correlations for the changes in caregiver burden and other variables at the 1-year follow-up

\begin{tabular}{lcc}
\hline & Baseline ZBI & Change ZBI \\
\hline K-MMSE & $-0.19^{\dagger}$ & -0.02 \\
Orientation for time & -0.09 & -0.06 \\
Orientation for place & -0.17 & -0.16 \\
Registration & -0.03 & 0.08 \\
Attention \& calculation & -0.16 & -0.02 \\
Recall & 0.35 & 0.02 \\
Language \& visual construction & $-0.20^{*}$ & 0.08 \\
S-ADL & 0.16 & 0.13 \\
Self-care/hygiene & $0.20^{*}$ & $0.22^{*}$ \\
Ambulation & -0.03 & -0.07 \\
Toileting & 0.16 & 0.05 \\
S-IADL & $0.26^{* *}$ & $0.25^{* *}$ \\
Non-memory-related functioning & $0.28^{* *}$ & 0.09 \\
Memory-related functioning & $0.20^{*}$ & $0.31^{* * *}$ \\
K-NPI & $0.37^{* * *}$ & $0.42^{* * *}$
\end{tabular}

${ }^{*} \mathrm{p}<0.05,{ }^{* *} \mathrm{p}<0.01,{ }^{* * *} \mathrm{p}<0.001,{ }^{\dagger} \mathrm{p}<0.06$. ZBI: Zarit Burden Interview, K-MMSE: Korean version-Mini Mental State Examination, S-ADL: Seoul-Activities of Daily Living, S-IADL: Seoul-Instrumental Activities of Daily Living, K-NPI: Korean version-Neuropsychiatric Inventory 


\section{Regression analyses}

Clinical variables of patients, with significant correlations with the ZBI total score were entered into the multiple regression analysis to identify variables that can best describe caregiver burden (Table 3). The total K-NPI score was a significant predictor of caregiver burden $[\beta=0.37, \mathrm{t}(108)=4.08, \mathrm{p}<0.001]$.

Changed clinical variable scores of patients, with significant correlations with the change in the ZBI score were entered into a multiple regression analysis to predict the change in caregiver burden (Table 3). The 1-year change in caregiver burden was predicted by the change in the total K-NPI score $[\beta=0.37$, $\mathrm{t}(107)=4.19, \mathrm{p}<0.001]$ and the S-IADL "memory-related functioning" factor $[\beta=0.22, t(107)=2.46, \mathrm{p}<0.05]$.

A multiple regression analysis was carried out at baseline to investigate which of the detailed behavioral problems can affect caregiver burden (Table 4). The total ZBI score was the dependent variable. Each of the K-NPI items that were significantly correlated with the ZBI score were independent variables. The following were found to be significant predictors affecting caregiver burden: K-NPI-irritability $[\beta=0.20, \mathrm{t}(105)=2.20$, $\mathrm{p}<0.05]$, K-NPI-aberrant motor behavior $[\beta=0.21, \mathrm{t}(105)=2.40$, $\mathrm{p}<0.05]$, K-NPI-delusions $[\beta=0.20, \mathrm{t}(105)=2.23, \mathrm{p}<0.05]$, and K-NPI-disinhibition $[\beta=0.20, t(105)=2.22, p<0.05]$. Additionally, the change in each of the K-NPI items scores, that had a significant association with the change in the ZBI total score, was entered into a multiple regression analysis (Table 4). As a result, K-NPI-delusions $[\beta=0.29, \mathrm{t}(107)=3.19, \mathrm{p}<0.01]$ and K-NPIagitation $[\beta=0.24, t(107)=2.63, p=0.01]$ were found to be able to significantly predict the total ZBI change score.

\section{DISCUSSION}

We investigated the relationships between caregiver burden and cognitive function, activities of daily-living, and neuropsychiatric symptoms in dementia patients. In particular, we examined the significant relationships between changes in variables of patients with dementia and changes in caregiver burden at the 1-year follow-up. Consistent with the majority of previous studies, ${ }^{7,8}$ we found that increased caregiver burden was steadily associated with patient neuropsychiatric symptoms at baseline and at the 1-year follow-up. Also, neuropsychiatric symptoms were the strongest predictor of caregiver burden at baseline and of changes between baseline and the 1-year follow-up. That is, neuropsychiatric symptoms were the most strongly associated factors with caregiver burden, suggesting that behavioral disturbances are the most important patient variable impacting caregiver burden. ${ }^{15}$

More specifically, our results show that delusions, disinhibition, irritability, and aberrant motor behavior were predictors of increased caregiver burden at the baseline. Additionally, delusions and agitated behavior increased caregiver burden at the 1-year follow-up. Noteworthy, our results also show that delusions are the persistent predictor of increased caregiver burden. Such neuropsychiatric symptoms, including delusions may be improved by pharmacotherapy to decrease caregiver burden. ${ }^{27}$ In addition, a symptom that is likely to resolve or per-

Table 3. Results of the multiple regression for caregiver burden at baseline and change in caregiver burden at the 1-year follow-up

\begin{tabular}{lccccc}
\hline & $\mathrm{R}^{2}$ & $\Delta \mathrm{R}^{2}$ & $\mathrm{~F}$ & $\beta$ & $\mathrm{t}$ \\
\hline Baseline & & & & & \\
$\quad$ & & & & \\
$\quad$ K-NPI & 0.133 & 0.125 & $16.63^{* * *}$ & & \\
1-year follow-up & & & & & \\
K-NPI & 0.177 & 0.169 & $23.18^{* * *}$ & 0.37 & $4.19^{* * *}$ \\
S-IADL memory-related functioning & 0.221 & 0.206 & $15.17^{* * *}$ & 0.22 & $2.46^{*}$ \\
\hline
\end{tabular}

${ }^{*} \mathrm{p}<0.05,{ }^{* * *} \mathrm{p}<0.001$. K-NPI: Korean version-Neuropsychiatric Inventory, S-IADL: Seoul-Instrumental Activities of Daily Living

Table 4. Results of the multiple regression for caregiver burden from the K-NPI and the detailed items at baseline and at the 1-year follow-up

\begin{tabular}{|c|c|c|c|c|c|}
\hline & $\mathrm{R}^{2}$ & $\Delta \mathrm{R}^{2}$ & $\mathrm{~F}$ & $\beta$ & $\mathrm{t}$ \\
\hline \multicolumn{6}{|l|}{ Baseline } \\
\hline K-NPI-irritability & 0.069 & 0.060 & $7.95^{* * *}$ & 0.20 & $2.20^{*}$ \\
\hline K-NPI-aberrant motor behavior & 0.129 & 0.113 & $7.92^{* * *}$ & 0.21 & $2.40^{*}$ \\
\hline K-NPI-delusions & 0.166 & 0.142 & $7.01^{* * *}$ & 0.20 & $2.23^{*}$ \\
\hline K-NPI-disinhibition & 0.203 & 0.173 & $6.70^{* * *}$ & 0.20 & $2.22 *$ \\
\hline \multicolumn{6}{|l|}{ 1-year follow-up } \\
\hline K-NPI-delusions & 0.140 & 0.132 & $17.58^{* * *}$ & 0.29 & $3.19^{* *}$ \\
\hline K-NPI-agitation/aggression & 0.192 & 0.177 & $12.72^{* * *}$ & 0.24 & $2.63^{*}$ \\
\hline
\end{tabular}

${ }^{*} \mathrm{p}<0.05,{ }^{* *} \mathrm{p}<0.01,{ }^{* * *} \mathrm{p}<0.001$. K-NPI: Korean version-Neuropsychiatric Inventory 
sist at a low level may best be addressed by simple behavioral interventions and caregiver support and education. ${ }^{28}$ In this respect, it is important to consider the type of symptom and its severity in understanding the burden of both the patient and caregiver. The recommended treatment intervention could be determined, at least in part, by symptom severity.

Several neuropsychiatric symptoms, such as delusions, disinhibition, irritability, and aberrant motor behavior, are reported to be linked to frontal lobe abnormalities. ${ }^{29}$ Agitation has also been strongly associated with functional disability and frontal-executive dysfunction across a range of dementia severity. Our findings suggest that interventions targeting a patient's behavioral symptoms could be an effective approach to decrease caregiver burden. Although our results are not applicable to all cases, clinical interventions with focus on controlling frontal-executive function may help to reduce caregiver burden. A previous study has suggested that frontal-executive dysfunction is an accurate predictor of caregiver burden. ${ }^{30}$ Thus, strategies to manage frontal-related behavioral problems, particularly executive dysfunction and disinhibition, will be important interventions to decrease caregiver burden. Mohamed et al. showed that treatment with antipsychotic drugs reduced psychiatric symptoms in patients with Alzheimer's disease, which significantly reduced caregiver burden. ${ }^{31}$

Memory-related daily-living function, such as keeping appointments, was not a predictor of caregiver burden at baseline but was at the 1-year follow-up. This may be due to the fact that a caregiver was more concerned about a patient's memory-related daily functions. As the memory impairment of patients became worse, it may have increased caregiver burden. However, patients basic daily-living function did not predict caregiver burden. Indeed, the number of patients with severe dementia was small. That is, 5 patients were rated as CDR 3 stage and they constitute a relatively small portion (4.5\%) of the total sample. Nevertheless, the cause of the null effect on caregiver burden should be identified, whether it is the small number of the CDR 3 stage in patients, or the effect of deteriorated memory per se. Meanwhile, the S-ADL scores ( $M=3.26$ at baseline) were lower than the average score, indicating that patients in this study did not have severe impairments in basic daily-living functions. Further studies are needed to verify the possible impact of dementia severity on daily-living functions.

A previous study suggests that language and poor communication, as well as impaired praxis, difficulties with coordination, and problems with spatial awareness contributed to caregiver burden. ${ }^{14}$ Deteriorating language and visual construction were associated with caregiver burden at baseline of our study. Although the literature is inconclusive about the relationship between caregiver burden and cognitive function in patients with dementia, ${ }^{9,12}$ we observed an association between lan- guage deterioration and visual construction and caregiver burden by separating the specific K-MMSE cognitive domains. Further study will be needed to verify which deteriorated cognitive function is an important factor affecting caregiver burden, and the reason why the cognitive function is associated with caregiver burden.

There exist a few important limitations in our study. Firstly, the current study did not measure possible factors that would yield systematic influence on caregiver burden, such as dementia severity, dementia type, age of onset (length of illness), or physical and psychiatric comorbidities. Further research would benefit from considering these factors, as it would enhance the generalizability of current findings. In addition, some studies have demonstrated the role of influence of family relationship between the patient and the caregiver on caregiver burden. ${ }^{32-34}$ However, the present study did not measure this factor (e.g., spouse, child, or other relatives). Thus, we suggest further study include relationship factor in their measures.

Secondly, patients and caregivers used in this study were from a specific clinical setting. Unfortunately, a considerable number of patients were lost to non-follow-up at 1-year, and the small sample size may also be classed as a limitation. Therefore, further study with samples from a regional community or various clinical settings might be needed. Additionally, even though our study was conducted as a longitudinal study and we acquired our data over 1 year, which is a relatively long time span compared with that of the previous study, extending the followup period would help to identify the long-term effects of patient symptoms in caregiver burden. Thirdly, although the present study shows that cognitive functioning based on the KMMSE was not a significant predictor of caregiver burden, more diverse cognitive assessments will be needed in order to build a broader comprehension of the relationship between cognitive deterioration and caregiver burden.

Although this study has some limitations, we have provided some implications for the relationships between caregiver burden and clinical variables of dementia patients, using our 1-year follow-up data. We used detailed factors or sub domains for each patient's clinical variable measurements (MMSE, S-ADL, S-IADL, and NPI) to verify the relationships between caregiver burden and dementia variables. Our results demonstrate which factor is the most significant predictor of caregiver burden among variables, and which factor could decrease caregiver burden. With our findings, we hope that more clinical research on caregiver burden will be conducted, so that caregivers of patients with dementia can receive more attention and appropriate intervention to decrease caregiver burden.

\section{Supplementary Materials}

The online-only Data Supplement is available with this ar- 
ticle at http://dx.doi.org/10.4306/pi.2016.13.1.43.

\section{Acknowledgments}

This study was supported by grants of the Korean Health Technology R\&D Project, Ministry of Health \& Welfare, Republic of Korea (HI14C2071). The funding bodies had no role in study design, data collection and analysis, decision to publish, or preparation of the manuscript.

\section{REFERENCES}

1. Dupuis SL, Epp T, Smale B. Caregivers of Persons with Dementia: Roles Experiences Supports and Coping. A Literature Review. Waterloo, ON: University of Waterloo; 2004.

2. Gwyther LP. Social issues of the Alzheimer's patient and family. Am J Med 1998;104:17S-21S; discussion 39S-42S.

3. Leinonen E, Korpisammal L, Pulkkinen LM, Pukuri T. The comparison of burden between caregiving spouses of depressive and demented patients. Int J Geriatr Psychiatry 2001;16:387-393.

4. Potter JF. Comprehensive geriatric assessment in the outpatient setting: population characteristics and factors influencing outcome. Exp Gerontol 1993;28:447-457.

5. Zarit SH, Todd PA, Zarit JM. Subjective burden of husbands and wives as caregivers: a longitudinal study. Gerontologist 1986;26:260-266.

6. Arai Y, Kumamoto K, Washio M, Ueda T, Miura H, Kudo K. Factors related to feelings of burden among caregivers looking after impaired elderly in Japan under the long-term care insurance system. Psychiatry Clin Neurosci 2004;58:396-402.

7. Clyburn LD, Stones MJ, Hadjistavropoulos T, Tuokko H. Predicting caregiver burden and depression in Alzheimer's disease. J Gerontol B Psychol Sci Soc Sci 2000;55:S2-S13.

8. Coen RF, Swanwick GR, O’Boyle CA, Coakley D. Behaviour disturbance and other predictors of carer burden in Alzheimer's disease. Int J Geriatr Psychiatry 1997;12:331-336.

9. Donaldson C, Burns A. Burden of Alzheimer's disease: helping the patient and caregiver. J Geriatr Psychiatry Neurol 1999;12:21-28.

10. Etters L, Goodall D, Harrison BE. Caregiver burden among dementia patient caregivers: a review of the literature. J Am Acad Nurse Pract 2008;20:423-428.

11. Rymer S, Salloway S, Norton L, Malloy P, Correia S, Monast D. Impaired awareness, behavior disturbance, and caregiver burden in Alzheimer disease. Alzheimer Dis Assoc Disord 2002;16:248-253.

12. Mangone CA, Sanguinetti RM, Baumann PD, Gonzalez RC, Pereyra S, Bozzola FG, et al. Influence of feelings of burden on the caregiver's perception of the patient's functional status. Dementia 1993;4:287-293.

13. Palley HA, Hanley BE, Watson D. Adult day care centers and the treatment of Alzheimer's disease patients: A critical analysis. Am J Alzheimer Dis Other Demen 1994;9:29-38.

14. Germain S, Adam S, Olivier C, Cash H, Ousset PJ, Andrieu S, et al. Does cognitive impairment influence burden in caregivers of patients with Alzheimer's disease? J Alzheimers Dis 2009;17:105-114.

15. Mohamed S, Rosenheck R, Lyketsos CG, Schneider LS. Caregiver burden in Alzheimer disease: cross-sectional and longitudinal patient correlates. Am J Geriatr Psychiatry 2010;18:917-927.

16. Folstein MF, Folstein SE, McHugh PR. "Mini-mental state". A practical method for grading the cognitive state of patients for the clinician. J Psy- chiatr Res 1975;12:189-198.

17. Ku HM, Kim JH, Kwon EJ, Kim SH, Lee HS, Ko HJ, et al. A study on the reliability and validity of Seoul-Instrumental Activities of Daily Living (S-IADL). J Korean Neuropsychiatr Assoc 2004;43:189-199.

18. Ku HM, Kim JH, Lee HS, Ko HJ, Kwon EJ, Jo S, et al. A study on the reliability and validity of Seoul-Activities of Daily Living (S-ADL). J Korean Geriatr Soc 2004;8:206-214.

19. American Psychiatric Association. Diagnostic and Statistical Manual of Mental Disorders: DSM-IV (4th Ed). Washington, DC: American Psychiatric Association; 1994.

20. McKhann G, Drachman D, Folstein M, Katzman R, Price D, Stadlan EM. Clinical diagnosis of Alzheimer's disease: report of the NINCDSADRDA Work Group under the auspices of Department of Health and Human Services Task Force on Alzheimer's Disease. Neurology 1984;34: 939-944.

21. Cohen CA, Colantonio A, Vernich L. Positive aspects of caregiving: rounding out the caregiver experience. Int J Geriatr Psychiatry 2002;17: 184-188.

22. Morris JC. Clinical Dementia Rating Scale. St. Louis: Washington University; 1994.

23. Kang Y, Na DL, Hahn S. A validity study on the Korean Mini-Mental State Examination (K-MMSE) in dementia patients. J Korean Neurol Assoc 1997;15:300-308.

24. Choi SH, Na DL, Kwon HM, Yoon SJ, Jeong JH, Ha CK. The Korean version of the neuropsychiatric inventory: a scoring tool for neuropsychiatric disturbance in dementia patients. J Korean Med Sci 2000;15: 609-615.

25. Cummings JL, Mega M, Gray K, Rosenberg-Thompson S, Carusi DA, Gornbein J. The Neuropsychiatric Inventory: comprehensive assessment of psychopathology in dementia. Neurology 1994;44:2308-2314.

26. Lee HS, Kim DK, Ko HJ, Ku HM, Kwon EJ, Kim JH. Measurement of stress in the caregivers of dementia patients: reliability and validity of the revised-memory and behavior problem checklist and the burden interview. Korean J Clin Psychol 2004;23:1029-1050.

27. Rabins PV, Lyketsos CG, Steele CD. Practical Dementia Care. New York: Oxford University Press; 1999.

28. Steinberg M, Tschanz JT, Corcoran C, Steffens DC, Norton MC, Lyketsos CG, et al. The persistence of neuropsychiatric symptoms in dementia: the Cache County Study. Int J Geriat Psychiatry 2004;19:19-26.

29. Senanarong V, Cummings JL, Fairbanks L, Mega M, Masterman DM, O'Connor SM, et al. Agitation in Alzheimer's disease is a manifestation of frontal lobe dysfunction. Dement Geriatr Cogn Disord 2004;17:14-20.

30. Davis JD, Tremont G. Impact of frontal systems behavioral functioning in dementia on caregiver burden. J Neuropsychiatry Clin Neurosci 2007; 19:43-49.

31. Mohamed S, Rosenheck R, Lyketsos CG, Kaczynski R, Sultzer DL, Schneider LS. Effect of second-generation antipsychotics on caregiver burden in Alzheimer's disease. J Clin Psychiatry 2012;73:121-128.

32. George LK, Gwyther LP. Caregiver well-being: a multidimensional examination of family caregivers of demented adults. Gerontologist 1986; 26:253-259.

33. Lund DA, Pett MA, Caserta MS. Institutionalizing dementia victims: some caregiver considerations. J Gerontol Soc Work 1987;11:119-135.

34. Cantor MH. Strain among caregivers: a study of experience in the United States. Gerontologist 1983;23:597-604. 